\title{
MEASUREMENTS OF IMPEDANCE DISTRIBUTIONS AND INSTABILITY THRESHOLDS IN LEP
}

\author{
D. Brandt, P. Castro, K. Cornelis, A. Hofmann, G. Morpurgo, G.L. Sabbi, J. Wenninger and B. Zotter, \\ CERN, CH-1211 Geneva 23, Switzerland
}

\begin{abstract}
The distribution of reactive and resistive components of the transverse impedance around the circumference of LEP has been obtained by exciting the beam and measuring the variations of phase advance and of closed orbit displacements with beam current. Tune shifts with current and instability thresholds were determined for two machine lattices with different beta functions and momentum compaction for various values of bunch length and synchrotron frequency. These measurements are particularly interesting for LEP2, which will require larger bunch currents to achieve the design luminosity.
\end{abstract}

\section{INTRODUCTION}

In the present operation of LEP near $45 \mathrm{Gev} /$ beam, the single bunch current is limited to less than about $0.6 \mathrm{~mA}$ both by the transverse mode coupling instability (TMCI) and by the beambeam effect. Due to the latter limitation, there is presently no advantage in increasing the single bunch current, and the way to reach higher luminosities is to use a larger number of bunches, such as is done in the "Pretzel" and "bunch-train" schemes. However, for LEP2, operating at higher energies by using superconducting cavities, the total beam current will be limited by synchrotron radiation losses which have to be compensated by the available RF power. Also the cryogenic system will provide a limited amount of cooling. On the other hand, the beambeam limit is expected to be higher in proportion to the energy of operation. Therefore it would be advantageous to operate with a small number of bunches with the highest current possible. For the standard scheme of 4 on 4 bunches, $1 \mathrm{~mA}$ per bunch would be necessary to obtain the desired design luminosity. In order to overcome limitations by collective effects, in particular to increase the TMCI threshold, a good understanding of all impedances in LEP is required. The impedances obtained by computer models should be verified on the machine. In addition to measurements of the total values of the impedances, it has now become possible to obtain individual contributions of structures situated around the circumference of LEP.

\section{CURRENT DEPENDENCE OF BETATRON PHASE ADVANCE}

\section{A. Introduction}

The reactive transverse impedance leads to a reduction of the betatron tunes with bunch current which has been measured for several machine lattices[1] in the past. In the present experiment it was tried to distinguish between the tune shift caused by the impedance of the RF-sections and that caused by the rest of the machine which is expected to be dominated by that of the numer- ous (shielded) bellows. This was done by measuring the betatron phase advance for different bunch currents using the "1000-turn" memory of the pickups[2].

\section{B. Machine Conditions for the First Experiment}

A positron beam was accumulated in the $90 / 60^{\circ}$ injection optics, with both damping and polarization wigglers excited. The horizontal and vertical phase advances as well as the tunes were measured for different bunch intensities using a continuous excitation of the betatron oscillation.

\section{Analysis of Measurements}

First, the tune dependence on the bunch current was measured with the Q-meter. An analysis of the results yielded

$$
\frac{d Q_{x}}{d I_{b}}=66 \mathrm{~A}^{-1}, \frac{d Q_{y}}{d I_{b}}=133 \mathrm{~A}^{-1} .
$$

All theoretical betatron phase advances were computed with the program MAD [3] and subtracted from the measurements. Several procedures were used to remove the ambiguity in absolute phase, such as considering only the difference in phase between the first and later monitors, or by subtracting the average phase of the first few monitors from the others for each current level. The measurements are presented as the change of the normalized phases per unit bunch current

$$
\frac{1}{2 \pi} \frac{d \mu_{x, y}}{d I_{b}}
$$

For further analysis two different methods were applied. The first one uses some physical insight into the observed effects in treating the results, while the second one makes least square fits through most of the data with as little bias as possible.

First method: To get easily measurable effects, the amplitudes of the betatron excitation were larger at low beam intensities than at higher ones. However, the analysis showed that phases measured with large amplitudes suffer from an additional non-linear detuning effect, which results in a rather poor correlation between those data and the ones taken at high intensity. For this reason, measurements with bunch currents below $200 \mu \mathrm{A}$ were eliminated from the analysis. The results obtained with the remaining data are presented in Table I and II, for both the vertical and the horizontal plane. As can be seen, the relative contributions of the bellows and the RF cavities are rather consistent for different sets of measurements. Moreover, at least in the vertical plane, the accumulated phase shift around the ring is in fair agreement with the values obtained directly with the Q-meter. However, a positive slope of the horizontal phase advance in some of the arcs remains unexplained and is thought to be produced 
by drifts of the machine parameters and inaccuracies of the measurements.

Second method: A least square fit was applied to all of the data taken in the experiment with the purpose of estimating the accuracy and in order to discover any errors caused by a possible bias in the treatment. Monitors which had very different readings from the adjacent ones were eliminated from the analysis (less than 20 out of a total of over 500). Results which had rather large values for all monitors, probably due to a phase off-set, were also eliminated. To remove ambiguities in the absolute values of the phase, a fit through the first octant was made to find the phase off-set at the beginning of the machine. To investigate the effect of the betatron oscillation amplitude on the measured phase advances, several runs with the same current but different excitations levels were compared.
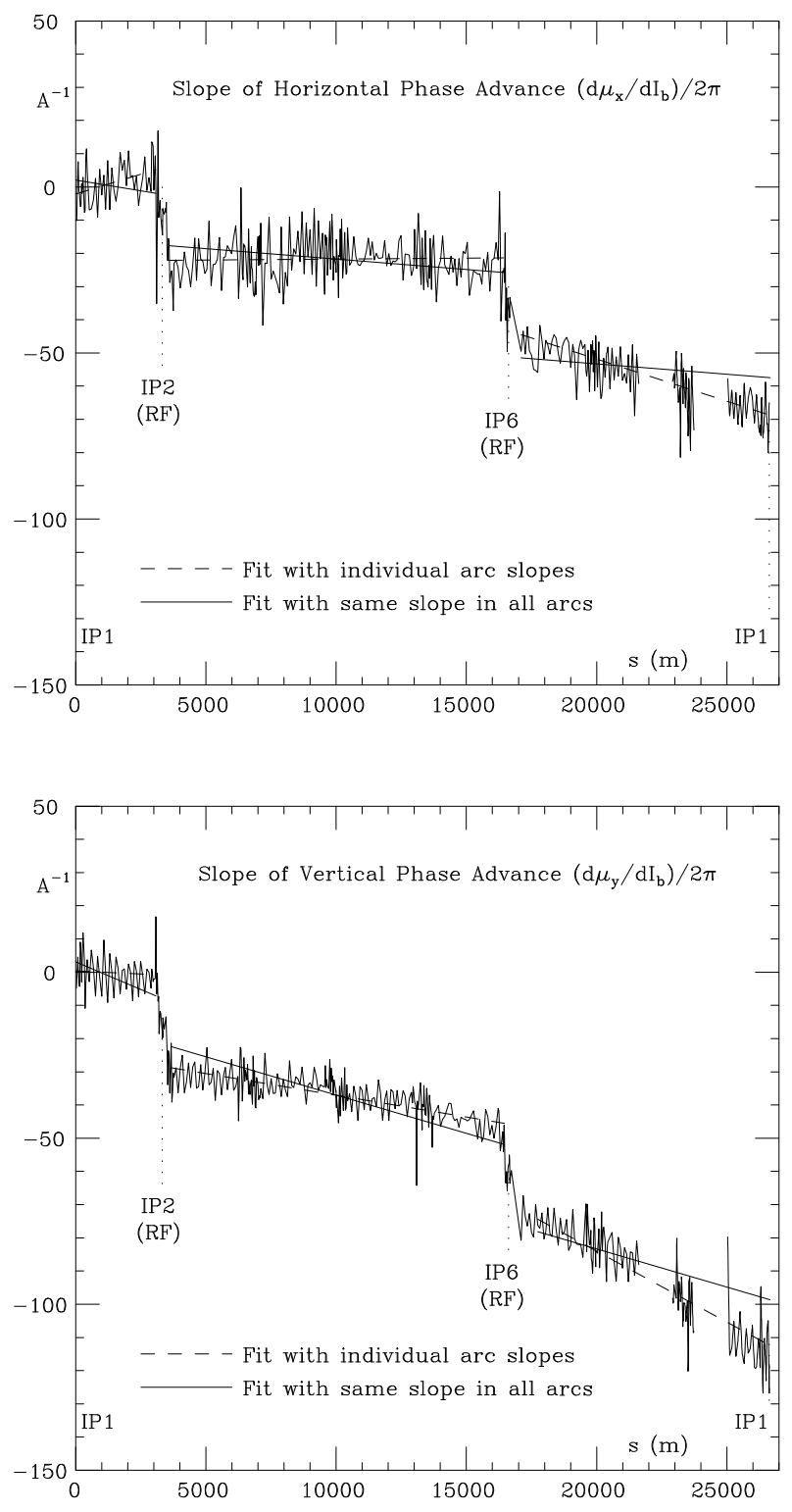

Figure. 1. Dependence of phase advances on bunch current

\begin{tabular}{|c|c|c|c|c|c|c|c|c|}
\hline$\left(\mathrm{A}^{-1}\right)$ & \multicolumn{3}{|c|}{ First analysis } & \multicolumn{3}{c|}{ Second analysis } & Average & Q-meter \\
\hline plane & arcs & cav. & total & arcs & cav. & total & total & total \\
\hline $\mathrm{x}$ & -13.1 & -54.3 & -67.5 & -16.7 & -40.5 & -57.2 & -62.4 & -66 \\
$\mathrm{y}$ & -63.8 & -59.5 & -123.4 & -64.7 & -49.7 & -114.4 & -118.9 & -133 \\
\hline
\end{tabular}

Table I

Measured phase advance per bunch current $d(\mu / 2 \pi) / d I_{b}$ for the RF cavities and the rest of LEP

The results of the second analysis are shown in Fig.1, obtained by linear fitting of measurements performed at 9 different bunch currents. The longitudinal distance $s$ from IP1 is used as horizontal coordinate. We see two large, localized impedances in the two RF-sections at IP2 and IP6. In the arcs, the slopes of individual sections were fitted with dashed lines. A slight variation of these slopes can be seen which may be caused by drifts of the machine parameters or measurement errors: all arcs should have about the same impedance, which is mainly caused by the large number (2800) of uniformly distributed (shielded) bellows. For this reason another fit was made with the same slope for all arcs as shown by the solid line. The fast oscillation of the phase is explained by a beta function mismatch, caused by the defocusing effect of the reactive impedance: this can be understood as the impedance acting as a current dependent quadrupole.

\section{Discussion}

In Table I, the slopes of the phase advance with current, obtained from the data with the two methods of analysis, are shown individually for the cavities and for the arcs. The averaged sums are compared with those of the direct measurement of the total tune shifts. From the scatter of the values obtained with the different methods we estimate an error about $20 \%$. To estimate the transverse impedance from these measurement, we normalized the phase advance per bunch current with the average beta functions as shown in Table II.

The tables show that the results have variations which depend on the way the fit is made. Measurements taken at large excitation amplitudes always had larger errors than those at smaller ones. In the second analysis, the total phase shifts around the ring were always smaller than the corresponding values obtained by direct measurement with the Q-meter.

\section{E. Comparison with Computation}

The effective transverse impedance is the integral over the machine impedance, multiplied by the bunch spectrum squared. For a particular impedance component, it can be found from the measured slopes of the phase shift by inverting the usual expression for the tune shift[4]

$$
Z_{T}^{e f f}=\frac{E_{o} \sigma_{s}}{e R\langle\beta\rangle} \frac{d \mu}{d I_{b}}
$$

The resulting values of the various contributions as well as the total effective transverse impedances are shown in Table.II.

From the definition, the effective transverse impedance depends on the bunch length. While it could not be measured in this experiment, it is known from previous experience to be approximately $\sigma_{s}=18-19 \mathrm{~mm}$ at injection energy when damping and polarization wigglers are excited and $Q_{s}=0.08$. The vertical impedance of LEP is dominated by the 120 five-cell copper 


\begin{tabular}{|c|c|c|c|c|c|c|c|}
\hline & & \multicolumn{2}{|c|}{ first analysis } & \multicolumn{2}{c|}{ second analysis } & \multicolumn{2}{c|}{ average } \\
\hline & units & arcs & cav. & arcs & cav. & arcs & cav. \\
\hline$\left\langle\beta_{x}\right\rangle$ & $\mathrm{m}$ & & & & & 64.3 & 39.3 \\
$\left\langle\beta_{y}\right\rangle$ & $\mathrm{m}$ & & & & & 84.9 & 40.7 \\
\hline$\frac{d \mu_{x} / d I_{b}}{2 \pi\left\langle\beta_{x}\right\rangle_{b}}$ & $(A m)^{-1}$ & -0.20 & -1.38 & -0.26 & -1.03 & -0.23 & -1.21 \\
$\frac{d \mu_{y} / d I_{b}}{2 \pi\left\langle\beta_{y}\right\rangle}$ & $(A m)^{-1}$ & -0.75 & -1.46 & -0.76 & -1.22 & -0.76 & -1.34 \\
\hline$Z_{x}^{\text {eff }}$ & $M \Omega / m$ & 0.11 & 0.76 & 0.14 & 0.57 & 0.13 & 0.67 \\
$Z_{y}^{\text {eff }}$ & $M \Omega / m$ & 0.41 & 0.80 & 0.42 & 0.67 & 0.42 & 0.74 \\
\hline$Z_{x}^{\text {eff }}$ & $M \Omega / m$ & & & & & $\leq 0.2$ & 0.65 \\
$Z_{y}^{\text {eff }}$ & $M \Omega / m$ & & & & & 0.40 & 0.65 \\
\hline
\end{tabular}

Table II

Normalized phase advance per bunch current divided by the average beta function and effective transverse impedances, measured and computed.

RF cavities $\left(Z_{y}=1.2 \mathrm{M} \Omega / m\right.$ at $\left.f_{\text {res }}=2 \mathrm{GHz}\right)$ and the 2800 shielded bellows $\left(Z_{y}=0.4 \mathrm{M} \Omega / m\right.$ at $\left.f_{\text {res }}=120 \mathrm{GHz}\right)$ [5], [6]. The horizontal impedance of the cavities with a circular beam hole should be same as the vertical one, while for the flat bellows it is estimated to be less than half that value. All other components (separators, pickup electrodes, collimators, etc.) contribute only about $10 \%$ in total [7] and are neglected.

For a Gaussian bunch in a broad-band resonator, the effective transverse impedance is given by the machine impedance $Z_{T}=R / Q$, multiplied with a reduction factor $F\left(\omega_{\text {res }} \sigma_{s} / c\right)$. The latter can be approximated by [4] $F(x)=2 x^{2}$ for very small $\mathrm{x}$, and approaches unity for $x \gg 1$. For the cavities the reduction factor is about $\mathrm{F}(0.8)=0.54$, while for the bellows it is near unity. The effective impedances for the two major impedances in LEP are shown at the bottom of Table II, and - considering the large errors in the phase shift data - compare quite well with the values obtained from the measurements.

\section{CURRENT DEPENDENCE OF THE ENERGY LOSS DISTRIBUTION}

\section{A. The Second Experiment}

By measuring the orbit displacement with current in pickups located in regions with finite values of dispersion, one can obtain the distribution of the energy loss around the machine circumference. In order to eliminate the effects of orbit distortions, only the differences between orbits at a very low bunch current $(50 \mu \mathrm{A})$ and 2 values of higher currents $(225$ and $500 \mu \mathrm{A})$ were measured. During the experiment, only one of the two RF sections (consisting of half the RF cavities) was powered, while the other one was idling (the results were very similar when the other $\mathrm{RF}$ section was powered). The data were averaged over each arc in order to eliminate the dependence of the orbit on spurious side effects. The results have been plotted and are shown in Fig.2.

\section{B. Comparison with Computation}

The resistive part of the effective longitudinal impedance, i.e. the longitudinal loss factor $k_{L}$, can be obtained from the energy loss as function of current. It depends strongly on bunch length which also in this experiment could not be measured. With only

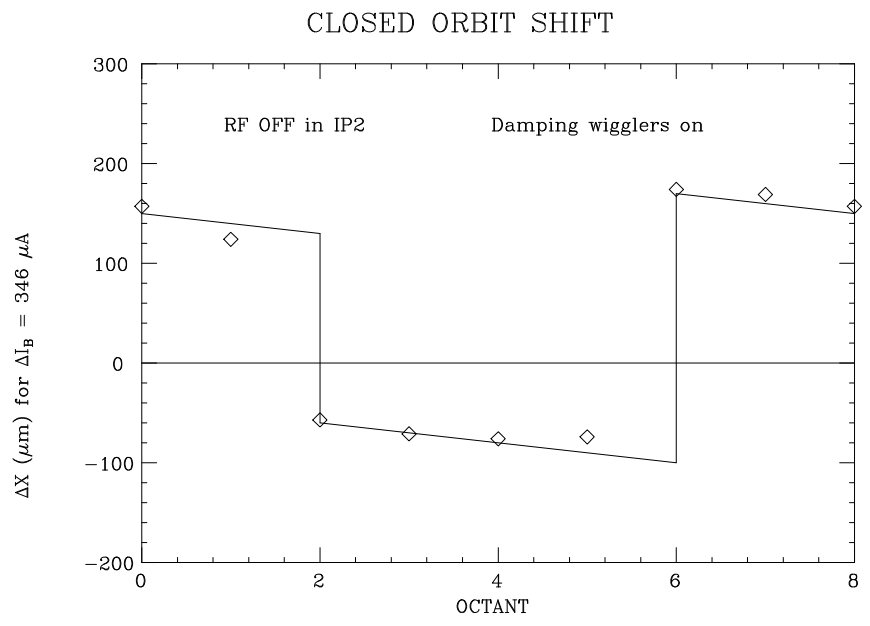

Figure. 2. Difference orbits for 2 values of current

the damping wigglers excited, the rms bunch length at injection energy $\left(E_{o}=20 \mathrm{GeV}\right)$ is about $10-11 \mathrm{~mm}$ for $Q_{s}=0.08$.

$$
k_{L}=\frac{E_{o} f_{o}}{e D_{x}} \frac{\Delta x_{c o}}{\Delta I_{b}}
$$

In the idling RF section, the data show an orbit displacement $\Delta x_{c o}=190 \mu \mathrm{m}$ for a current increase $\Delta I_{b}=342 \mu \mathrm{A}$. With an average dispersion $D_{x}=0.58 \mathrm{~m}$ in the pickups, and the revolution frequency $f_{0}=11.25 \mathrm{KHz}$ in LEP, this expression yields a loss factor of about $210 \mathrm{~V} / \mathrm{pC}$. For a bunch length of $11 \mathrm{~mm}$, the computed loss factor of an RF cavity cell is about $0.58 \mathrm{~V} / \mathrm{pC}$, or $185 \mathrm{~V} / \mathrm{pC}$ for one section consisting of 64 five-cell cavities. The rest must be due to all other elements in the RF straight section. The total orbit displacement in both arcs is about $80 \mu \mathrm{m}$, corresponding to a loss factor of about $90 \mathrm{~V} / \mathrm{pC}$. For $\sigma_{s}=10 \mathrm{~mm}$, one shielded bellows unit has a loss factor of about $8 \mathrm{~V} / \mathrm{nC}$, yielding about $22 \mathrm{~V} / \mathrm{pC}$ for 2800 of them. The loss factor for the resistive wall effect is given by

$$
k_{L}=0.612 \omega_{o} Z_{o} \delta_{o} /\langle b\rangle\left(R_{a r c} / \sigma_{s}\right)^{3 / 2}
$$

With $R_{\text {are }}=4.1 \mathrm{~km}, \delta_{o}=0.81 \mathrm{~mm}$ and $\langle b\rangle=45.6 \mathrm{~mm}$ we get $k_{L}=60 \mathrm{~V} / \mathrm{pC}$. The total is quite close to the measured value. Considering the uncertainties in bunch length and shape, the agreement is very satisfactory.

\section{References}

[1] D. Brandt et al.: "The LEP Impedance Model", Proc. of 1993 Particle Accelerator Conference; p.3429 (1993).

[2] J. Borer et al.: "Harmonic Analysis of Coherent Bunch Oscillations in LEP", Proc. EPAC, Berlin 1992, p.1082

[3] H. Grote, F. Iselin "The Mad Program"; CERN SL/90-13.

[4] LEP Commissioning Notes 21 (1989) and 75 (1990)

[5] B. Zotter: "Comparison of Theory and Experiment on Beam Impedances, Proc. EPAC, Berlin 1992, p.273

[6] B. Zotter: "Collective Effects", Proc. 3rd LEP Performance Workshop, Chamonix 1993, CERN SL/93-19, p.89

[7] G. Sabbi: "Simulation of Single-Bunch Collective Effects in LEP”, CERN SL/94 report in preparation 